Results TP bacteria were mainly localised in the epithelial sheets, even if some TP were also detected in perivascular areas of the dermis. The infiltrate was characterised, as expected, by large number of plasmocytes, detected using MAbs anti- CD138, CD79a and MUM-1. Plasmocytes showed active in situ proliferation, as demonstrated by MIB-1/Ki-67 proliferation marker. An abundant epidermotropic T-lymphocytes infiltrate consisting of the CD8+ subset was easily demonstrated in the superficial dermis, where several macrophages (M1 and M2) were also detected. Unexpectedly the staining of dendritic cells by using anti CD1a MAb evidenced the quite complete absence of Langerhans cells, both in the epidermis and in the dermis.

Conclusions Our results, showing that syphilis infiltrate is mainly composed by abundant plasmocytes and CD8+ lymphocytes in absence of dendritic Langerhans cells, suggest the presence of a characteristic immune-disregulation in the skin affected from TP infections. Further studies on a larger number of patients are needed in order to better clarify the exact immunological mechanisms in skin syphilitic lesions.

\section{P1.008 INTRASTRAIN GENETIC HETEROGENEITY IN TREPONEMA PALLIDUM SSP. PALLIDUM}

doi:10.1136/sextrans-2013-051184.0229

D Cejkova, M Strouhal, D Smajs. Masaryk University Brno, Brno, Czech Republic

Background Next-generation sequencing techniques, besides determination of complete genome sequences and interstrain variability, can assess variability within individual bacterial strains. Here we examined intrastrain heterogeneity in two strains of T. pallidum ssp. pallidum, the agent of syphilis.

Methods To ascertain intrastrain heterogeneity within Nichols and SS14 strains, whole-genome sequencing Illumina reads were used. Individual reads were mapped to complete genomes using Borrows-Wheeler Aligner. To determine the allele frequency of mapped reads for every genome position, the SAMtools package and Perl script were applied and heterogenous positions showing at least six independent reads calling for an alternative allele were further examined. To distinguish true variants from sequencing errors, each heterogenous position was visually evaluated.

Results Altogether, 17 and 31 intrastrain variable positions were found in the unique regions of Nichols and SS14 genomes, respectively. Only 2 positions in each strain were located in intergenic regions. The variable positions of the Nichols and SS14 strains altered 16 and 29 genes, respectively, while four genes (coding for cell division protein, TprB protein, polymerase A and putative lipoprotein) were altered in both genomes at the same positions. Generally, genes for hypothetical proteins and putative membrane proteins, and transporters showed intrastrain heterogeneity in both genomes. Moreover, for the SS14 strain, variable positions in genes coding for DNA repair mechanism and motility were found.

Conclusion The identified variable regions of treponemal genomes appear to provide a selective advantage in host-pathogen interaction. To determine the extent of intrastrain heterogeneity in other pathogenic treponemes, additional completed genomes will be evaluated.

\section{P1.009 ANALYSIS OF SIMPLE SEQUENCE REPEATS IN THE GENOMES OF PATHOGENIC TREPONEMAL STRAINS}

doi:10.1136/sextrans-2013-051184.0230

'M Strouhal, ${ }^{1} \mathrm{M}$ Zobanikova, ${ }^{1} \mathrm{D}$ Cejkova, ${ }^{1} \mathrm{H}$ Petrosova, ${ }^{1} \mathrm{P}$ Pospisilova, ${ }^{1} \mathrm{~B}$ Staudova, ${ }^{2} \mathrm{G}$ M Weinstock, 'D Smajs. 'Masaryk University Brno, Brno, Czech Republic; '2Washington University in St. Louis, Saint Louis, MO, United States

Background Simple sequence repeats (SSR) are repetitive sequences of 1-6 base pairs in DNA which account for genotypic switching (phenotypic variation) through mechanism of polymerase slippage. This mechanism is common in pathogenic bacteria with reduced genome.

Methods Msatfinder v2.0.9 was used to identify SSR sequences in the genomes of five strains of Treponema pallidum subsp. pallidum (agent of syphilis); three strains of T. $p$. subsp. pertenue (agent of yaws); the Bosnia A strain of T.p. subsp. endemicum (agent of endemic syphilis); the Cuniculi A strain of T. paraluiscuniculi (agent of rabbit syphilis); and the Fribourg-Blanc strain (simian isolate genetically close to agent of yaws). Analysis of Solexa data and subsequent cloning approach were used for determination of intrastrain variability.

Results Sequence data analysis of 11 treponemal genomes revealed high degree of variability in guanosine/cytosine homopolymeric tracts (G/C-regions) among pathogenic treponemal strains. Altogether, $120 \mathrm{G} / \mathrm{C}$-regions (containing $>7$ nucleotides) were found, from which 53 regions showed no variability, 26 represented nucleotide substitution differences, and 41 contained variable numbers of $\mathrm{G} / \mathrm{Cs}$. From these 41 regions, 25 were located in intergenic regions and 16 affected ORFs. Interestingly, variable regions were located upstream or within genes coding for Tpr proteins, potential virulence factors, and hypothetical proteins. Furthermore, 30 regions were specific for Cuniculi A strain, 5 regions differentiated pallidum strains from other strains, and 3 regions were specific for pertenue strains. Moreover, additional variability within treponemal strains was found in 54 G/C-regions.

Conclusions Inter- and intrastrain variability of G/C-regions may play a significant role in pathogenesis of treponemal diseases. Further experimental studies are needed to verify this hypothesis.

\section{P1.010 COMPARATIVE EVOLUTIONARY ANALYSES OF NINE TREPONEMA PALLIDUM AND TREPONEMA PARALUISCUNICULI STRAINS}

doi:10.1136/sextrans-2013-051184.0231

\section{P Krecmerova, D Smajs. Masaryk University Brno, Brno, Czech Republic}

Background Pathogenic uncultivable treponemes include the agents of syphilis (T. pallidum ssp. pallidum, TPA), yaws (T. pallidum ssp. pertenue, TPE) and endemic syphilis (T. pallidum ssp. endemicum, TEN). Treponema paraluiscuniculi (TPC) causes rabbit syphilis. Pathogenic treponemes are highly clonal bacteria showing minimal genetic variability in the genome sequence of individual strains.

Methods Five TPA strains (Nichols, SS14, Chicago, Mexico A and DAL-1), three TPE strains (CDC-2, Samoa D and Gauthier) and a one TPC strain Cuniculi A were analysed in this study. All possible combinations of gene alignments were tested on type of selection by Z-test using modified Nei-Gojobori method based on Jukes-Cantor model. Complete deletion was used as a gap treatment and transition/transversion ratio was set to 0.85 . Results were considered significant at $5 \%$ level.

Results A total of 22 genes were found under positive selection in at least one comparison between treponemal strains. Negative and neutral selection was found in at least one combination for 258 and 206 genes, respectively. While positive selection was identified in genes encoding putative virulence factors, proteins involved in cell structure and cell processes, negative selection was predominantly found in genes encoding components of general metabolism, transport and translation. Neutral selection was detected mainly in genes encoding hypothetical proteins and genes encoding proteins involved in general metabolism.

Conclusions Positively selected genes are candidates for important treponemal virulence factors while negatively selected and conserved genes are likely to encode essential genes. Genes under neutral evolution may indicate genome regions that could be lost during adaptation of pathogen to its host. 